\title{
Descent for quasi-coherent sheaves on stacks
}

\author{
SHARON HOLLANDER
}

\begin{abstract}
We give a homotopy theoretic characterization of sheaves on a stack and, more generally, a presheaf of groupoids on an arbitary small site $\mathfrak{C}$. We use this to prove homotopy invariance and generalized descent statements for categories of sheaves and quasi-coherent sheaves. As a corollary we obtain an alternate proof of a generalized change of rings theorem of Hovey.
\end{abstract}

14A20; 18G55, 55U10

\section{Introduction}

Stacks were introduced in order to parametrize problems in algebraic geometry where the presence of automorphisms prevented representability by a scheme or even a sheaf (see Artin [1], Deligne-Mumford [3] and Giraud [5]). One early application was Deligne and Mumford's use of stacks to prove the irreducibility of the space of curves of a given genus [3]. More recently stacks have also played an important role in algebraic topology. Complex oriented cohomology theories give rise to stacks over the moduli stack of formal groups and, in certain situations, stacks over the moduli stack of formal groups give rise to spectra (see Goerss [6], Goerss-Hopkins [8] and Rezk [21]) which play an important role in understanding the homotopy groups of spheres (see Goerss-Henn-Mahowald-Rezk [7] and Behrens [2]). One fundamental example is the spectrum of topological modular forms (see Hopkins [12]) which is associated to the moduli stack of elliptic curves.

The purpose of this paper is to continue the study of stacks from the point of view of homotopy theory. We generalize basic definitions and constructions pertaining to (quasi-coherent) sheaves on stacks, to an arbitrary small site 5 and an arbitrary presheaf of groupoids on 5 (not just a stack). We use this point of view to give new proofs of fundamental theorems in this setting. In the rest of the introduction we will give a general overview of these results; precise definitions will be given in Sections 2 and 3 .

Classically, stacks are defined as those categories fibered in groupoids over $\mathfrak{S}$, (or equivalently lax presheaves of groupoids on 5 ) which satisfy descent [3, Definition 4.1]. 
In [11] we show that a category fibered in groupoids $F$ over $\mathbb{C}$ is a stack if and only if the assignment satisfies the homotopy sheaf condition, that is, for each cover $\left\{U_{i} \rightarrow X \in \mathfrak{S}\right\}$, the natural map

$$
F(X) \stackrel{\sim}{\longrightarrow} \operatorname{holim}\left(\prod F\left(U_{i}\right) \Longrightarrow \prod F\left(U_{i j}\right) \Longrightarrow \prod F\left(U_{i j k}\right) \cdots\right)
$$

is an equivalence of categories, (where the homotopy limit here is taken in the category of small groupoids, denoted (Srpd, which is a simplicial model category).

This characterization of stacks naturally leads to a model structure on categories fibered in groupoids over $\mathfrak{E}$, in which the fibrant objects are the stacks. Similarly, one can consider the strict functors, or presheaves of groupoids on $\mathbb{5}$, denoted $P(\mathfrak{S}$, (Srpd). Here too there is a local model structure, denoted $P(\mathfrak{S} \text {, (كrpd })_{L}$, in which the fibrant objects are those functors which are stacks or, equivalently, satisfy the homotopy sheaf condition. Furthermore, there is a Quillen equivalence between these two model categories. (See [11, Section 4]).

Since this paper will derive results about sheaves on stacks from the ambient model category it makes no difference which of the Quillen equivalent model categories one chooses to work in. For the sake of simplicity we will work in $P(\widetilde{S}, \text { (Srpd })_{L}$.

Given a stack $\mathcal{M}$, on $\mathfrak{E}$, the category of sheaves on $\mathcal{M}[3$, Definition 4.10] is defined as sheaves on the site $\widetilde{S} / \mathcal{M}$. The site $\subseteq / \mathcal{M}$ can be easily generalized to an arbitrary presheaf of groupoids $\mathcal{M}$ and site $\mathfrak{S}$ (see Section 2.1). Here objects of $\mathfrak{S} / \mathcal{M}$ are morphisms $X \rightarrow \mathcal{M} \in P(\mathfrak{S}$, (Jrpd), with $X \in \mathfrak{S}$, and the morphisms are triangles with a commuting homotopy. Covers in $\mathbb{E} / \mathcal{M}$ are the collections of morphisms which forget to covers in $\mathfrak{C}$. Notice that the underlying category $\widetilde{S} / \mathcal{M}$ is just the Grothendieck construction on the functor $\mathcal{M}$ : $\mathfrak{S}^{\circ} \rightarrow$ Grpd. Also notice that if $\mathcal{M}$ is represented by an object $X \in \mathfrak{C}$ this is the usual topology on the over category $\mathfrak{E} / X$.

We prove that the category of sheaves on $\sqrt{5} / \mathcal{M}$ is equivalent to the full subcategory of fibrations $F \rightarrow \mathcal{M}$ in $P\left(\mathcal{S}_{\text {, (S } r p d)_{L}}\right.$ where the fibers $F(X) \rightarrow \mathcal{M}(X)$ are discrete for each $X \in \mathfrak{C}$. In fact, this yields an embedding of sheaves on $\mathcal{M}$ as a full subcategory of the homotopy category $\operatorname{Ho}\left(P(\mathfrak{C} \text {, (Srpd })_{L} / \mathcal{M}\right)$ (Corollary 4-6).

Using this embedding we prove that a local weak equivalence $\mathcal{M} \rightarrow \mathcal{M}^{\prime}$ induces via the restriction functor an equivalence of categories

$$
\operatorname{Sh}\left(\widetilde{C} / \mathcal{M}^{\prime}\right) \rightarrow \operatorname{Sh}(\widetilde{S} / \mathcal{M})
$$

(Theorem 4-7). This also holds for sheaves of abelian groups, simplicial sets, rings, modules. 
We also present a definition of a quasi-coherent sheaf of modules over a sheaf of rings $\mathcal{O}$ in an arbitrary site, (see Definition 4-11), and show that the results of the previous paragraph holds for quasi-coherent sheaves (Corollary 4-10).

Classically, sheaves on an algebraic stack $\mathcal{M}$ are described via an atlas. If $X \rightarrow \mathcal{M}$ an atlas, sheaves on $\mathcal{M}$ can be written as a sheaves on $X$, with an isomorphism of the two pullbacks to $X \times{ }_{\mathcal{M}} X$ satisfying the cocycle condition (see for instance Laumon-Moret-Bailly [14, Lemme 12.2.1]).

We generalize this and prove the following descent statement: given an $I$-diagram $\mathcal{M}_{I}$ in $P(\mathfrak{C}$, (Srpd) there is an equivalence of categories

$$
\operatorname{Sh}\left(\mathfrak{S} /\left(\operatorname{hocolim} \mathcal{M}_{i}\right)\right) \rightarrow \operatorname{holim} \operatorname{Sh}\left(\mathfrak{S} / \mathcal{M}_{i}\right)
$$

(where the homotopy limit is taken in 5 at with the categorical model structure, see Rezk [20]). The same holds for sheaves taking values in any category with products (Proposition 5-5). We prove also the analog for quasi-coherent sheaves of modules (Proposition 5-9). We think of the diagram $\mathcal{M}_{I}$ as a generalized presentation of hocolim $\mathcal{M}_{I}$.

Our descent statement generalizes the classical scenario since (by [10, Proposition A.9]) given an atlas $X \rightarrow \mathcal{M}$, the induced map below is a weak equivalence

$$
\operatorname{hocolim}\left(\cdots X \times_{\mathcal{M}}^{h} X \times_{\mathcal{M}}^{h} X \Longrightarrow X \times_{\mathcal{M}}^{h} X \Longrightarrow X\right) \stackrel{\sim}{\longrightarrow} \mathcal{M},
$$

and so it follows that $\operatorname{Sh}(\mathfrak{S} / \mathcal{M})$ is the homotopy inverse limit of the categories

$$
\operatorname{Sh}(X) \Longrightarrow \operatorname{Sh}\left(X \times_{\mathcal{M}}^{h} X\right) \Longrightarrow \operatorname{Sh}\left(X \times_{\mathcal{M}}^{h} X \times_{\mathcal{M}}^{h} X\right) \ldots
$$

which is a modern formulation of the classical statement written above (see Section 4.1).

A simple application of this descent statement (Proposition 5-15) implies that the category of comodules over an Hopf algebroid $(A, \Gamma)$ is equivalent to the category of quasicoherent sheaves on the presheaf of groupoids represented by the pair ( $\operatorname{Spec} A, \operatorname{Spec} \Gamma$ ) and so is also equivalent to quasicoherent sheaves on its stackification $\mathcal{M}_{(A, \Gamma)}$ (which is its fibrant replacement in $P\left(\mathfrak{A} / f f_{\text {flat }},\left(\operatorname{Srpd}_{L}\right)\right.$.

It follows that if $(A, \Gamma)$ and $\left(B, \Gamma^{\prime}\right)$ are two weakly equivalent Hopf algebroids then the categories of comodules on each are equivalent (Corollary 5-16).

The greater generality here is important for many reasons. First we provide an elementary description of the category of sheaves on a stack which is independent of the choice of site and makes sense for any stack $\mathcal{M}$ algebraic or not (compare with 
Laumon-Moret-Bailly [14, Chapter 12]). The descent statement shows that alternative descriptions of the category of sheaves on $\mathcal{M}$ can be obtained in many fashions, not just via an atlas, and not only in geometric contexts. In particular, in the case of algebraic stacks these description are not a by-product of the geometry but of category theory. Enlarging one's frame of reference to include presheaves of groupoids which are not stacks also enlarges the range of presentations and so the possible alternative descriptions of one's category of sheaves.

The moduli stack of formal groups $\mathcal{M}_{F G}$ is of special importance in stable homotopy theory (see Goerss [6], Pribble [18] and Naumann [17]). The Lazard ring provides an atlas $\operatorname{Spec} L \rightarrow \mathcal{M}_{F G}$. But $L$ is not noetherian and the maps $\operatorname{Spec} L \times_{\mathcal{M}_{F G}} \operatorname{Spec} L \rightarrow$ Spec $L$ are not finitely presented. It follows that $\mathcal{M}_{F G}$ is not an algebraic stack and much of the classical literature concerning sheaves on a stack does not apply.

We believe that in the context of problems whose origin is homotopy theory, larger classes of presentations for stacks should naturally appear and our descent statements will be of use.

Finally, as in [11], we believe that the proper context in which to understand stacks is a homotopy theoretic one. Weak equivalences (or 2-equivalences) of stacks are not homotopy equivalences. One can not work in a naive homotopy category of stacks and behave as if these equivalences were isomorphisms and the 2-category pullback were a real pullback. A model category structure provides a powerful way in which to contextualize these equivalences and the constructions that take them into account. Abstract homotopy theory was invented precisely to solve these types of problems.

\subsection{Relation to other work}

Part of the results here are bringing those of Hovey [13] into the homotopy theoretic framework of [11]. In [13], Hovey defines quasi-coherent sheaves on a presheaf of groupoids on $\mathfrak{A} f f_{\text {flat }}$ and prove a generalized change of rings theorem. It is a consequence of Corollary 4-6 and Lemma 5-11 that our definition of (quasi-coherent) sheaves agrees with [13, Definitions 1.1 and 1.2]. Our Proposition 5-15 then is exactly Theorem A in [13]. [11, Proposition 5.7] implies that the internal equivalences of [13, Definition 3.1] agree with our local weak equivalences. It follows that Propositions 4-7 and 4-10 are exactly Theorems B and C of [13], when the site $\subseteq=\mathfrak{S} f f_{\text {flat }}$. [13, Theorem D] also follows directly from [11, Proposition 5.7].

\subsection{Acknowledgements}

The project of understanding stacks from the point of view of homotopy theory was inspired by a course by M Hopkins at M.I.T. and his ideas permeate this work. I would 
also like to thank G Granja, H Miller, and F Neumann for many helpful comments. This research was partially supported by the Center for Mathematical Analysis, Geometry, and Dynamical Systems at the Instituto Superior Técnico of the Technical University of Lisbon and the Golda Meir Fellowship Trust at the Hebrew University of Jerusalem.

\section{Background}

In this section we recall some results from the homotopy theory of categories, groupoids and presheaves of groupoids from Rezk [20] and from [11] and in the course of this fix our notation and conventions for the rest of the paper.

\subsection{Homotopy theory of categories}

Recall that Grpd has a cofibrantly generated simplicial model category structure in which:

- weak equivalences are equivalences of categories, and

- fibrations are maps $p: G \rightarrow H$ such that given $\alpha: b \stackrel{\sim}{\longrightarrow} p(a) \in H$ there exists $\beta: c \rightarrow a \in G$ with $p(\beta)=\alpha$.

- the simplicial structure is inherited via the fundamental groupoid functor $\pi_{\text {oid }}$.

- The generating trivial cofibration is $* \rightarrow \pi_{\mathrm{oid}} \Delta^{1}$. The generating cofibrations are $\{*, *\} \rightarrow \pi_{\mathrm{oid}} \Delta^{1}, B \mathbb{Z} \rightarrow *, \varnothing \rightarrow *$.

There is also a simplicial model category structure on 5 at in which weak equivalences are equivalences of categories and fibrations are the maps which have the right lifting property with respect to $* \rightarrow \pi_{\mathrm{oid}} \Delta^{1}$. The simplicial structure on 5 at is defined by setting $\mathfrak{5} \otimes X=\mathfrak{5} \times \pi_{\text {oid }} X$ and $\mathfrak{5}^{X}=\mathfrak{\complement} a t\left(\pi_{\text {oid }} X\right.$, $\left.\mathfrak{5}\right)$. For more details see [20]. We will sometimes abuse notation and write $\Delta^{1}$ for $\pi_{\text {oid }} \Delta^{1}$.

It follows from Hirschhorn $[9,18.1 .2,18.1 .8,18.5 .3]$ that we have the following explicit formulas for homotopy limits and colimits in 5 at. The homotopy inverse limit of an $I$ diagram of categories $\mathfrak{S}_{I}$ is the equalizer

$$
\prod_{o b(I)} \subseteq(i)^{\pi_{\mathrm{oid}}(I / i)} \rightrightarrows \prod_{i \rightarrow j \in I} \subseteq(j)^{\pi_{\mathrm{oid}}(I / i)}
$$

which can also be described as the end of the functors $\complement^{(}(-)$and $\pi_{\text {oid }}(I /-)$, see $[9$, 18.3]. Similarly the homotopy colimit of the diagram is the coequalizer

$$
\coprod_{i \rightarrow j \in I} \mathfrak{S}(i) \times \pi_{\text {oid }}(j / I) \rightrightarrows \coprod_{i \in I} \subseteq(i) \times \pi_{\text {oid }}(i / I)
$$

Algebraic $8 \mathcal{G}$ Geometric Topology, Volume 7 (2007) 
or the coend $\widetilde{S}(-) \otimes_{I} \pi_{\text {oid }}(-/ I)$.

We can also compute the homotopy (co)limit by taking a cosimplicial (simplicial) replacement of our diagram and applying the Tot ("geometric realization") functor.

As the simplicial structure on Sat derives from a (Srpd enrichment, it follows from [11, Theorems 2.9 and 2.12] that Tot is equivalent to $\operatorname{Tot}^{2}$. The category $\operatorname{Tot}^{2}\left(\mathfrak{C}^{\bullet}\right)$ of a cosimplicial category $\mathfrak{5}^{\bullet}$, has

- objects pairs $(x, \alpha)$ where $x$ is an object of $\mathbb{5}^{0}$ and $\alpha: d^{0} x \stackrel{\sim}{\longrightarrow} d^{1} x$ is an isomorphism in $\mathfrak{C}^{1}$ satisfying $s^{0} \alpha=\mathrm{id}_{x}$ and $d^{2} \alpha \circ d^{0} \alpha=d^{1} \alpha$, and

- morphisms $(x, \alpha) \rightarrow(y, \beta)$ consist of $h: x \rightarrow y \in \mathfrak{S}^{0}$ such that $\beta \circ d^{0} h=$ $d^{1} h \circ f$.

Using cosimplicial replacement one obtains from this formula a compact description of an arbitrary homotopy limit.

Similarly a model for the homotopy colimit of a simplicial diagram of categories $\widetilde{\zeta}_{\bullet}$ is the coend in $\mathfrak{S}_{a}$,, $\mathfrak{S}_{\bullet} \otimes_{\Delta} \pi_{\text {oid }} \Delta[-]$, which we also refer to as the geometric realization, denoted $\left|\mathfrak{S}_{\bullet}\right|$. Here too we have a smaller model for $\left|\mathfrak{S}_{\bullet}\right|$ where the objects are the objects of $\mathfrak{S}_{0}$ and the morphisms are generated by those in $\mathfrak{S}_{0}$ and the isomorphisms $f_{y}: d_{0} y \rightarrow d_{1} y$ for each $y \in \mathfrak{S}_{1}$, subject to the relations

- $f_{s_{0} x}=\mathrm{id}_{x}$

- for $y \stackrel{g}{\longrightarrow} y^{\prime} \in \mathfrak{S}_{1}, d_{1} g \circ f_{y}=f_{y^{\prime}} \circ d_{0} g$, and

- for $z \in \mathfrak{S}_{2}, f_{d_{2} z} \circ f_{d_{0} z}=f_{d_{1} z}$.

The formulas above also give descriptions of homotopy (co)limits in Srpd (note that the inclusion of (Srpd in (5at preserves limits and colimits).

\subsection{Sites and presheaves}

We will always assume that our sites $\widetilde{C}$ are small and closed under finite products and pullbacks. We write $P(\mathfrak{S}, \mathfrak{D})$ for the category of presheaves on $\mathbb{S}$ with values in a category $\mathfrak{D}$ and $P(\mathfrak{S})=P(\mathfrak{C}$, كet $)$. We abuse notation and identify the objects in $\mathbb{5}$ with the presheaves of sets (or discrete groupoids) they represent.

If $\left\{U_{i} \rightarrow X\right\}$ is a cover, we write $U=\bigsqcup_{i} U_{i}$ for the coproduct of the presheaves and $F(U)$ for $\operatorname{Hom}(U, F)=\prod_{i} F\left(U_{i}\right) . U_{\bullet}$ is the nerve of the cover which is the simplicial object obtained by taking iterated fiber products over $X$. We will sometimes abuse notation and write a cover as $U \rightarrow X .\left|U_{\bullet}\right|$ will denote the geometric realization of the simplicial object in $P(\mathfrak{S}$, (Srpd). Recall that the geometric realization of a simplicial 
diagram $F_{\bullet}$ in $P\left(\right.$ (ᄃ, (Śrpd) is defined by $\left|F_{\bullet}\right|(Y)=\left|F_{\bullet}(Y)\right|$ (see [11, Section 2.2]). In particular, $\left|U_{\bullet}\right|(Y)$ is the groupoid whose objects are $\bigsqcup_{i} \operatorname{Hom}\left(Y, U_{i}\right)$ and whose isomorphisms are generated by the set $\bigsqcup_{i, j} \operatorname{Hom}\left(Y, U_{i} \times_{X} U_{j}\right)$ satisfying the obvious relations (see the previous subsection).

We will consider two different model structures on the category of presheaves of groupoids. $P\left(\mathfrak{S}\right.$, (Srpd) will denote the levelwise model structure where a map $F \rightarrow F^{\prime}$ is a fibration (weak equivalence) if and only if $F(X) \rightarrow F^{\prime}(X)$ is a fibration (weak equivalence) in (Sirpd. We will write $P(\mathfrak{S} \text {, (Srpd })_{L}$ for the local model structure which is the localization of $P(5$, ( $r p d)$ with respect to the maps

$$
\left\{\left|U_{\bullet}\right| \rightarrow X \in P(\text { (ᄃ, (śrpd })\right\}
$$

where $\left\{U_{i} \rightarrow X\right\}$ is a cover in (5. $F \in P(\mathcal{C}, \text { (Srpd })_{L}$ is fibrant iff $F(X) \rightarrow \operatorname{holim}_{\Delta} F\left(U_{\bullet}\right)$ is an equivalence of groupoids for all covers $\left\{U_{i} \rightarrow X\right\}$, that is, iff $F$ is a stack (see [11]). The stack condition is a direct generalization of the sheaf condition since a presheaf of sets $F$ is a sheaf if and only if $F(X) \rightarrow \lim _{\Delta} F\left(U_{\bullet}\right)$ is an isomorphism for all covers $\left\{U_{i} \rightarrow X\right\}$.

Note that, by the definition of localization, the cofibrations and trivial fibrations in $P\left(\mathfrak{S},(\text { S } r p d)_{L}\right.$ are the same as those in $P(\mathfrak{S}$, (كrpd). Unless otherwise noted, when we say a map of presheaves of groupoids $F \rightarrow G$ is a fibration or weak equivalence we mean in the local model structure.

$P(\mathfrak{S}$, Grpd) is enriched with tensor and cotensor over Grpd in the obvious way and therefore also over $s$ ऽet. Moreover, with this enrichment $P\left(\mathfrak{S}\right.$, (Srpd) and $P(\mathfrak{S} \text {, (Srpd })_{L}$ are simplicial model categories (see [11]).

Note that the geometric realization of a simplicial groupoid can be constructed by a finite sequence of pushouts along cofibrations and so $\left|F_{\bullet}\right|$ is cofibrant in $P(\mathfrak{C}$, (Sr rpd) so long as $F_{0}, F_{1}$ and $F_{2}$ are. In particular, if $\left\{U_{i} \rightarrow X\right\}$ is a cover, $\left|U_{\bullet}\right|$ is always cofibrant.

We say that a levelwise fibration $F \rightarrow F^{\prime}$ in $P\left(\mathcal{C}^{5}\right.$, (Jrpd) has discrete fibers if for each $X \in \mathfrak{C}$ the fiber of $F(X) \rightarrow F^{\prime}(X)$ over each object $a \in F^{\prime}(X)$ is a discrete groupoid (that is, a groupoid with only identity morphisms).

$\operatorname{Hom}_{P(\mathfrak{S},(\mathfrak{G r p d})}(F, G)$ denotes the groupoid of maps between two presheaves of groupoids. We write $\operatorname{hHom}(A, B)$ for the homotopy function complex of maps between two objects $A$ and $B$ in a model category.

We will use repeatedly the following basic result [11, Theorem 5.7] characterizing the weak equivalences in $P(\mathfrak{S} \text {, ( or } r p d)_{L}$ as those maps $F \stackrel{\phi}{\longrightarrow} G \in P(\mathfrak{S}$, (Srpd) which satisfy the local lifting conditions: 
(1) Given a commutative square

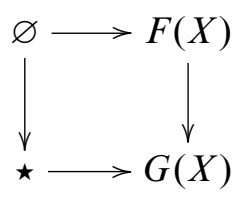

there exists a cover $U \rightarrow X$ and lifts in the diagram as follows:

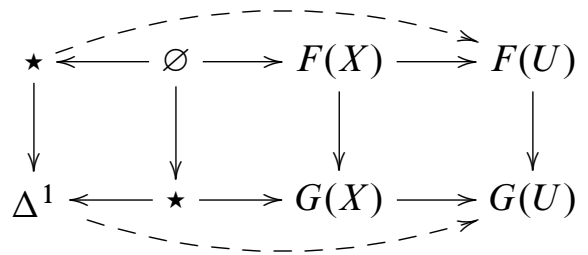

(2) For $A \rightarrow B$, one of the generating cofibrations in (Srpd (see [11, Section 2.1]) $\partial \Delta^{1}=\{\star, \star\} \rightarrow \Delta^{1}$ or $B \mathbb{Z} \rightarrow \star$, given a commutative square

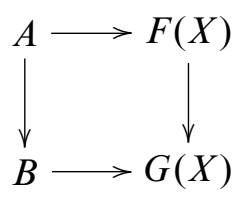

there exists a cover $U \rightarrow X$ and a lift in the diagram as follows:

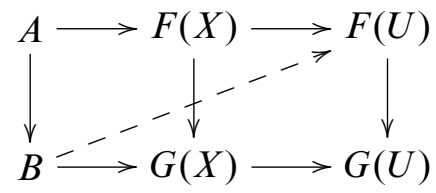

Note that condition (1) means that $F \rightarrow G$ is locally essentially surjective while condition (2) says that $F \rightarrow G$ is locally full and faithful.

\section{Presheaves on a Stack}

In this section we associate a site to a presheaf of groupoids $\mathcal{M}$ and prove an equivalence of categories between presheaves of groupoids on this site and the full subcategory $\left(P(\widetilde{S},(\text { Srpd }) / \mathcal{M})_{d f}\right.$ of the over category consisting of levelwise fibrations with discrete fiber.

Algebraic $8 \mathcal{G}$ Geometric Topology, Volume 7 (2007) 


\subsection{Grothendieck topology on $\mathcal{M}$}

The site we define is a simple generalization of the one first defined by Deligne and Mumford in [3, Definition 4.10].

Definition 3-1 Let $\mathcal{M}$ be presheaf of groupoids on $\sqrt{5}$ and let $5 / \mathcal{M}$ denote the category whose

- objects are pairs $(X, f)$ where $X \in \mathfrak{S}$ and $X \stackrel{f}{\longrightarrow} \mathcal{M} \in P(\mathfrak{C}$, (Srpd),

- morphisms from $X \stackrel{f}{\longrightarrow} \mathcal{M}$ to $X^{\prime} \stackrel{g}{\longrightarrow} \mathcal{M}$ are pairs $(h, \alpha)$ where $X \stackrel{h}{\longrightarrow} X^{\prime}$ and $\alpha$ is a homotopy $f \rightarrow g \circ h$.

Remark 3-2 (a) Given maps $f, f^{\prime}: X \rightarrow \mathcal{M}$, a homotopy $\alpha: f \rightarrow f^{\prime}$ determines an isomorphism in $\subseteq / \mathcal{M}$ between the objects $f$ and $f^{\prime}$ and so a presheaf $F$ on $\subseteq / \mathcal{M}$ will satisfy $F(X, f) \cong F\left(X, f^{\prime}\right)$.

(b) The category $5 / \mathcal{M}$ is just the Grothendieck construction on the functor $\mathcal{M}$, that is, the category whose objects are pairs $(X, a)$ with $X \in \mathfrak{S}$ and $a \in \mathcal{M}(X)$ and morphisms defined in the obvious way.

Notice that the projection $\mathfrak{S} / \mathcal{M} \rightarrow \mathfrak{S}$ is $p \mathcal{M}$, the category fibered in groupoids associated to $\mathcal{M}$ [11, Definition 3.11].

Lemma 3-3 Let $(g, \alpha):\left(Y, g^{\prime}\right) \rightarrow(X, f)$ and $(h, \beta):\left(Z, h^{\prime}\right) \rightarrow(X, f)$ be maps in $5 / \mathcal{M}$. The pullback of the maps $(g, \alpha)$ and $(h, \beta)$ in $5 / \mathcal{M}$ is

$$
\left(Y \times{ }_{X} Z, f \circ(g \times h)\right)
$$

where $g \times h$ denotes the canonical map $Y \times_{X} Z \stackrel{g \times h}{\longrightarrow} X$. The projection maps are $\left(p_{Y}, \alpha^{-1}\right)$ and $\left(p_{Z}, \beta^{-1}\right)$.

Using the previous lemma, the proof of the following proposition is an easy exercise.

Proposition 3-4 Let $\mathbb{C}$ be a site and $\mathcal{M} \in P(\widetilde{C}$, ( $r r p d)$. The collections of morphisms which forget to covers in $\mathbb{5}$ form the basis for a Grothendieck topology on $\mathbb{5} / \mathcal{M}$.

Remark 3-5 The site of Proposition 3-4 generalizes the étale site [3, 4.10] of a Deligne-Mumford stack $\mathcal{M}$ which has

- objects the schemes étale over $\mathcal{M}$, and

- morphisms triangles with a commuting homotopy, and

- covers those morphisms which forget to étale covers. 
The Deligne-Mumford site is $5 / \mathcal{M}$ when we take $\sqrt{5}$ to be the category of schemes and étale maps between them. If we take $\widetilde{S}$ to be the category of schemes and all maps between them, and $\mathcal{M}$ to be the sheaf represented by a scheme, the site of Proposition 3-4 is strictly bigger. It is called the big étale site of $\mathcal{M}$ (see Tamme [22, II.3.3]).

The sites that arise through the construction above are always over categories. This rules out some examples such as the smooth-étale site of an algebraic stack (see Laumon-Moret-Bailly [14, Definition 12.1]).

Definition 3-6 Let $T$ be a site. We say a collection of covers $S$ generates the topology on $T$ when a presheaf $F$ on $T$ is a sheaf if and only if it satisfies the sheaf condition when applied to a cover in $S$.

Proposition 3-7 The collection of covers of the form

$$
\left\{\left(U_{i}, f \circ u_{i}\right) \stackrel{\left(u_{i}, \mathrm{id}\right)}{\longrightarrow}(X, f)\right\}
$$

generate the topology on $\subseteq / \mathcal{M}$.

Proof Consider an arbitrary cover $\left\{\left(U_{i}, f_{i}\right) \stackrel{\left(u_{i}, \alpha_{i}\right)}{\longrightarrow}(X, f)\right\}$. We may factor these maps as

$$
\left(U_{i}, f_{i}\right) \stackrel{\left(\mathrm{id}, \alpha_{i}\right)}{\longrightarrow}\left(U_{i}, f \circ u_{i}\right) \stackrel{\left(u_{i}, \mathrm{id}\right)}{\longrightarrow}(X, f) .
$$

The first map is an isomorphism. If $F$ is a presheaf, the sheaf condition applied to the original cover requires that the top row in the following diagram be an equalizer while the sheaf condition applied to $\left\{\left(U_{i}, f \circ u_{i}\right) \stackrel{\left(u_{i}, \text { id }\right)}{\longrightarrow}(X, f)\right\}$ requires that the bottom row be an equalizer.

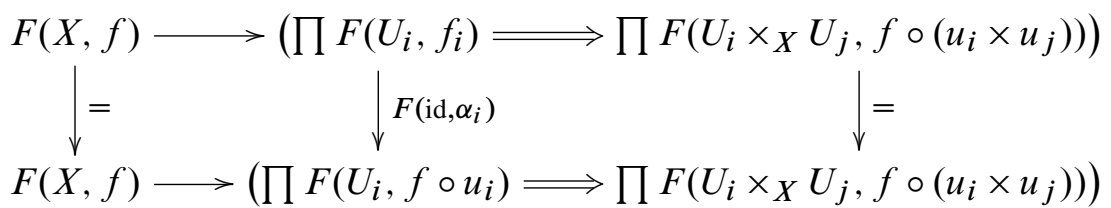

Since the above diagram commutes these two conditions are equivalent.

\subsection{Presheaves on $\mathcal{M}$}

We will now define an equivalence of categories between $P(\mathfrak{S} / \mathcal{M})$ and the full subcategory of $P(\widetilde{C}$, (Srpd) $/ \mathcal{M}$ consisting of levelwise fibrations with discrete fibers, which we denote by $(P(\widetilde{S}$, (rispd $) / \mathcal{M}) d f$.

Algebraic 83 Geometric Topology, Volume 7 (2007) 
Definition 3-8 Given $G \in P(\widetilde{S} / \mathcal{M})$, let $B G \in P(\mathfrak{S}$, (Srpd) be the presheaf so that $B G(X)$ is the groupoid whose objects are pairs $(a, s)$ where $a \in \mathcal{M}(X)$ and $s \in G(X, a)$. A morphism $(a, s) \rightarrow\left(b, s^{\prime}\right)$ is a morphism $a \stackrel{\alpha}{\longrightarrow} b \in \mathcal{M}(X)$ such that $s=\alpha^{*} s^{\prime}$.

Alternatively, $B G(X)$ is the Grothendieck construction on the restriction of the functor $G$ to the subcategory $\mathcal{M}(X)$ of $\subseteq / \mathcal{M}$. The proof of the following lemma is an easy exercise.

Lemma 3-9 The natural projection $B G \rightarrow \mathcal{M}$ is a levelwise fibration with discrete fibers. Moreover the fiber in $B G(X)$ over $a \in \mathcal{M}(X)$ is the set $G(X, a)$.

It is easy to check that $B$ defines a functor from $P(\widetilde{S} / \mathcal{M})$ to $(P(\widetilde{S}, \text { (srpd }) / \mathcal{M})_{d f}$.

A fibration $\pi: G \rightarrow H$ of groupoids with discrete fibers satisfies unique path lifting and so the assignment $a \mapsto \pi^{-1}(a)$ for $a \in \mathrm{ob} H$ defines a functor from $H^{\mathrm{op}}$ to Set. Using this it is easy to see that the following definition makes sense.

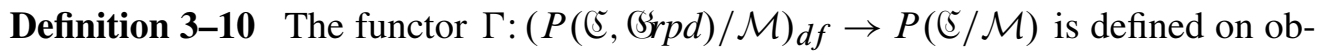
jects by $\Gamma(F \stackrel{\pi}{\longrightarrow} \mathcal{M})(X, a)=\pi_{X}^{-1}(a)$ where $\pi_{X}^{-1}(a)$ denotes the fiber in $F(X)$ over $a \in \mathcal{M}(X)$.

Proposition 3-11 The pair $(B, \Gamma)$ is an adjoint equivalence of categories.

Proof There is a natural isomorphism $G \rightarrow \Gamma B G$ which when evaluated at $(X, a)$ sends an element $s \in G(X, a)$ to the element $(a, s)$ in $B G(X)$ lying over $a$. Given $H \in(P(\widetilde{S}, \text { Grpd }) / \mathcal{M})_{d f}$, an element of $B \Gamma H(X)$ is a pair $(a, s)$ where $a \in \mathcal{M}(X)$ and $s$ is in the fiber of $H(X)$ over $a$. Sending $(a, s)$ to $s \in H(X)$ defines a natural isomorphism $B \Gamma H \rightarrow H$ over $\mathcal{M}$.

\section{Sheaves}

In this section we identify sheaves on $\mathcal{M}$ with the category of fibrations $\mathfrak{N} \rightarrow \mathcal{M}$ in $P(\widetilde{S}, \text { (Srpd })_{L}$ with objectwise discrete fiber, which we denote by $\left(P(\widetilde{S}, \text { (Srpd })_{L} / \mathcal{M}\right)_{d f}$. This homotopy theoretic characterization of the sheaves on a presheaf of groupoids allows us to prove invariance under weak equivalence. We also extend these results to the categories of sheaves of rings and sheaves of quasi-coherent modules.

Proposition 4-1 The functors $(B, \Gamma)$ restrict to give an equivalence of categories between $\operatorname{Sh}(\subseteq / \mathcal{M})$ and $\left(P(\mathfrak{S}, \operatorname{Sr} r p d)_{L} / \mathcal{M}\right)_{d f}$. 
Proof By Proposition 3-7 a presheaf $F$ on $\subseteq / \mathcal{M}$ is a sheaf if and only if

$$
F(X, a) \cong \text { equalizer }\left(\prod F\left(U_{i}, a \circ u_{i}\right) \rightrightarrows \prod F\left(U_{i j}, a \circ u_{i j}\right)\right)
$$

for all covers $\left\{U_{i} \stackrel{u_{i}}{\longrightarrow} X\right\}$ and $a \in \mathcal{M}(X)$. Since

$$
F(X, a)=\operatorname{Hom}_{P(\mathfrak{E},(\zeta r p d) / \mathcal{M}}(X \stackrel{a}{\longrightarrow} \mathcal{M}, B F \rightarrow \mathcal{M})
$$

the sheaf condition can be rewritten as

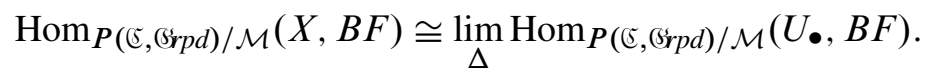

As $B F \rightarrow \mathcal{M}$ has discrete fibers, each groupoid of maps into it is discrete and therefore the inverse limit of $\operatorname{Hom}_{P\left(\mathbb{E},\left(G_{r} p d\right) / \mathcal{M}\right.}\left(U_{\bullet}, B F\right)$ agrees with the homotopy inverse limit.

This shows that $F$ is a sheaf if and only if $B F \rightarrow \mathcal{M}$ is local with respect to the maps $\left|U_{\bullet}\right| \rightarrow X \in P\left(\mathfrak{S}\right.$, (Srpd) $/ \mathcal{M}$ where $U_{\bullet}$ is the nerve of a cover of $X$. It follows from the following Proposition that this is equivalent to $B F \rightarrow \mathcal{M}$ being a fibration.

Proposition 4-2 A map $\widetilde{\Im} \rightarrow \mathcal{M}$ in $P\left(\widetilde{S} \text {, ( } \mathfrak{S}^{r} \mathrm{rd}\right)_{L}$ is a fibration if and only if it is a levelwise fibration and satisfies descent for covers, meaning for all covers $\left\{U_{i} \rightarrow X\right\}$ in $\mathbb{5}$, the following is a homotopy pullback square:

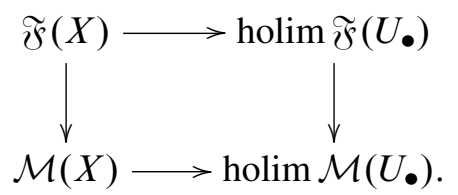

Proof The outline of the proof follows the arguments in Dugger-Hollander-Isaksen [4, Lemmas 7.2 and 7.3, Proposition 7.3].

Let $A \rightarrow B$ denote a generating cofibration $\varnothing \rightarrow *, B \mathbb{Z} \rightarrow *,\{*, *\} \rightarrow \Delta^{1}$ in Srrpd. Let $J$ be the set of morphisms in $P(\mathcal{C}$, (Sirpd) consisting of

$$
Z \rightarrow Z \times \Delta^{1}, Z \in \mathfrak{C}
$$

and

$$
\left|U_{\bullet}\right| \times B \coprod_{\left|U_{\bullet}\right| \times A} \tilde{X} \times A \rightarrow \tilde{X} \times B
$$

where $\left\{U_{i} \rightarrow X\right\}$ is a cover and $\left|U_{\bullet}\right| \rightarrow \tilde{X} \rightarrow X$ is the factorization of the natural map into a cofibration followed by a trivial fibration in $P\left(\mathcal{S}^{\text {, ( S r }} \mathrm{rpd}\right)_{L}$.

We claim that a map $\widetilde{\Im} \rightarrow \mathcal{M}$ has the right lifting property with respect to the morphisms in $J$ iff it satisfies descent for covers and is a levelwise fibration. First note that a 
map $\mathfrak{\Im} \rightarrow \mathcal{M}$ is a levelwise fibration iff it has the right lifting property with respect to the maps $\left\{Z \rightarrow Z \times \Delta^{1}\right\}$. Next observe that for a levelwise fibration $\mathfrak{\mho} \rightarrow \mathcal{M}$, the canonical map

$$
\operatorname{Hom}(\tilde{X}, \widetilde{\Im}) \longrightarrow \operatorname{Hom}\left(\left|U_{\bullet}\right|, \widetilde{\Im}\right) \times_{\operatorname{Hom}\left(\left|U_{\bullet}\right|, \mathcal{M}\right)} \operatorname{Hom}(\tilde{X}, \mathcal{M})
$$

is a fibration (because $\left|U_{\bullet}\right| \rightarrow \tilde{X}$ is a cofibration in the levelwise model structure $P(\mathfrak{S},($ Srpd $)$ ). A levelwise fibration $\mathfrak{\Im} \rightarrow \mathcal{M}$ satisfies the right lifting property with respect to $J$ if and only if the map (4-3) is a trivial fibration, and therefore if and only if the following square is homotopy cartesian

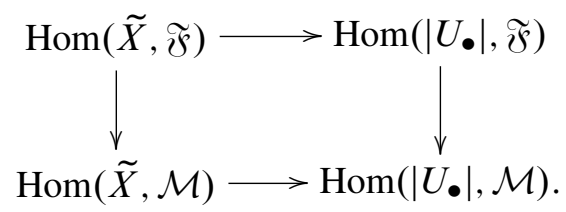

By definition, $\tilde{X} \rightarrow X$ is a trivial fibration and therefore a levelwise weak equivalence. Since $X$ and $\tilde{X}$ are cofibrant and all objects are levelwise fibrant,

$$
\operatorname{Hom}(X, \mathfrak{F}) \rightarrow \operatorname{Hom}(\tilde{X}, \mathfrak{F}), \quad \operatorname{Hom}(X, \mathcal{M}) \rightarrow \operatorname{Hom}(\tilde{X}, \mathcal{M})
$$

are weak equivalences. This completes the proof of the claim.

It now suffices to show that $J$ provides a set of generating trivial cofibrations for $P\left(\mathfrak{S},(\text { Grpd })_{L}\right.$. By [4, Lemma 7.3] it is enough to show that if $\mathfrak{\Im} \rightarrow \mathcal{M}$ is a weak equivalence, a levelwise fibration, and satisfies descent for covers then it is in fact a levelwise trivial fibration. We'll check the right lifting property of $\mathfrak{F}(X) \rightarrow \mathcal{M}(X)$ with respect to the generating cofibrations in Grpd for every $X \in \mathfrak{E}$.

Given a diagram

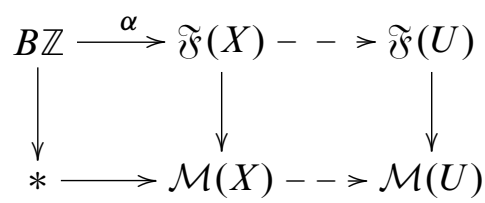

there exists a cover $U \rightarrow X$ such that the isomorphism $\alpha$ becomes trivial in $\mathfrak{\wp}(U)$. As holim $\mathfrak{F}\left(U_{\bullet}\right) \rightarrow \mathfrak{F}(U)$ is faithful, it follows that $\alpha$ is also trivial in holim $\mathfrak{F}\left(U_{\bullet}\right)$. As $\mathfrak{F} \rightarrow \mathcal{M}$ satisfies descent for covers, $\alpha$ must be trivial to begin with. This shows that $\mathfrak{\Im}(X) \rightarrow \mathcal{M}(X)$ is faithful for all $X$. 
Given a commutative square

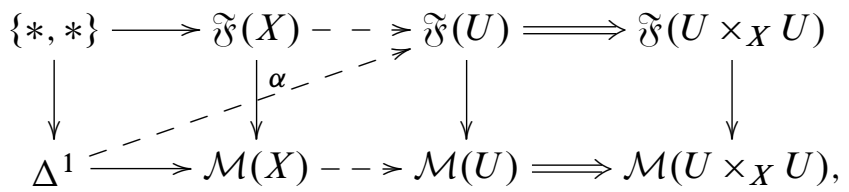

the local lifting conditions provide us with a lift $\alpha$ and the two images of $\alpha$ in $\mathfrak{F}\left(U \times{ }_{X}\right.$ $U$ ) lie over the same morphism in $\mathcal{M}\left(U \times_{X} U\right)$. Since $\mathfrak{\wp} \rightarrow \mathcal{M}$ is levelwise faithful it follows that $\alpha$ is equalized by the two maps. Thus $\alpha$ gives rise to a morphism in holim $\mathfrak{r}\left(U_{\bullet}\right)$ and hence in $\mathfrak{r}(X)$. Thus $\mathfrak{r}(X) \rightarrow \mathcal{M}(X)$ is also full.

Given $a \in \mathcal{M}(X)$, the local lifting conditions provide us with a cover $U \rightarrow X$ such that $a$ lifts to an element in $\mathfrak{F}(U)$. As the two images in $\mathcal{M}\left(U \times_{X} U\right)$ are isomorphic, levelwise fullness implies that they are isomorphic in $\mathfrak{\Im}\left(U \times{ }_{X} U\right)$ and this isomorphism satisfies descent by faithfulness of the map $\mathfrak{F}\left(U \times_{X} U \times_{X} U\right) \rightarrow \mathcal{M}\left(U \times_{X} U \times_{X} U\right)$. This provides us with an element in $\operatorname{holim} \mathfrak{r}\left(U_{\bullet}\right)$ and as $\widetilde{\mathfrak{r}} \rightarrow \mathcal{M}$ satisfies descent for covers, this element lifts to $\mathfrak{\Im}(X)$ up to isomorphism. This proves essentially surjectivity of $\mathfrak{F}(X) \rightarrow \mathcal{M}(X)$ and completes the proof.

Corollary 4-4 Given levelwise fibrations $\mathfrak{\wp} \stackrel{\sim}{\longrightarrow} \mathfrak{F}^{\prime} \rightarrow \mathcal{M}$ with the first map a levelwise weak equivalence, then $\widetilde{r}^{\prime} \rightarrow \mathcal{M}$ is a fibration if and only if $\widetilde{\wp} \rightarrow \mathcal{M}$ is a fibration.

Proposition 4-5 If $G$ is a sheaf on $\mathcal{M}$ and $H \in P(\widetilde{S} \text {, (srpd })_{L} / \mathcal{M}$ then

$$
\operatorname{Hom}_{P(\mathfrak{s},(\mathfrak{s} r p d) / \mathcal{M}}(H, B G) \cong \operatorname{hHom}_{P\left(\mathfrak{S},(\mathfrak{s} p d)_{L} / \mathcal{M}\right.}(H, B G)=[H, B G]_{P\left(\mathfrak{S},(\mathfrak{s} r p d)_{L} / \mathcal{M}\right.}
$$

Proof Let $K$ denote the cofibrant replacement of $H$ in $P(\mathbb{S}$, (Jrpd). We need to show that $\operatorname{Hom}_{P(\mathfrak{S},(\mathfrak{G r p d}) / \mathcal{M}}(K, B G) \cong \operatorname{Hom}_{P(\mathfrak{S}, \text { Grpd }) / \mathcal{M}}(H, B G)$. Given $X \in \mathfrak{S}$, the map $K(X) \rightarrow H(X)$ is a trivial fibration, so writing $K(X)_{a}$ for the fiber over $a \in$ ob $H(X)$, we have a pushout square in Grpd

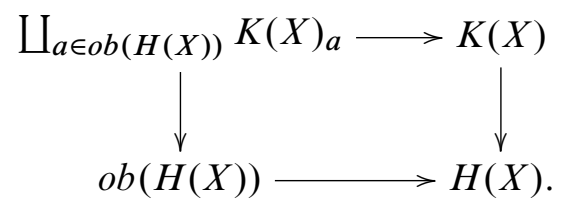

Algebraic 83 Geometric Topology, Volume 7 (2007) 
Since the fibers of $G(X) \rightarrow \mathcal{M}(X)$ are discrete, it follows that there is a unique extension

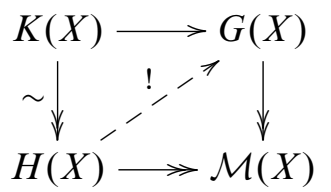

so every map from $K$ factors uniquely through $H$. This proves the first equivalence in the statement.

On the other hand, since $B G \rightarrow \mathcal{M}$ has discrete fibers, two maps $K \rightarrow B G \in$ $P(\mathfrak{S}$, (srpd) $/ \mathcal{M}$ are homotopic if and only if they are equal, which completes the proof.

If $F, F^{\prime}$ are presheaves on $\mathcal{M}, \operatorname{Hom}_{P\left(\mathfrak{S},\left(\zeta^{\prime} p d\right) / \mathcal{M}\right.}\left(B F, B F^{\prime}\right)$ is a discrete groupoid so we have the following corollary.

Corollary 4-6 The composition

$$
\operatorname{Sh}(\mathfrak{S} / \mathcal{M}) \stackrel{B}{\longrightarrow} P(\mathfrak{S}, \text { (orpd })_{L} / \mathcal{M} \rightarrow H o\left(P(\mathfrak{S}, \text { (كrpd })_{L} / \mathcal{M}\right)
$$

induces an equivalence of $\operatorname{Sh}(\mathfrak{S} / \mathcal{M})$ with the full subcategory of the homotopy category which consists of fibrant objects with levelwise discrete fiber.

We can now prove the main result of this section which states the invariance of the categories of sheaves under a local equivalence of presheaves of groupoids.

Theorem 4-7 $A$ weak equivalence $\mathcal{M}^{\prime} \stackrel{p}{\longrightarrow} \mathcal{M}$ in $P(\mathfrak{S} \text {, (Sírpd })_{L}$ induces a Quillen equivalence

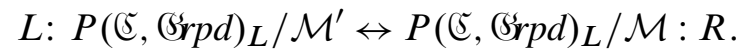

The induced equivalence of homotopy categories yields an equivalence of categories

$$
p_{*}: \operatorname{Sh}\left(\widetilde{C} / \mathcal{M}^{\prime}\right) \leftrightarrow \operatorname{Sh}(\widetilde{C} / \mathcal{M}): p^{*}
$$

where the right adjoint $p^{*}$ is composition with $\overleftarrow{5} / \mathcal{M}^{\prime} \stackrel{p}{\longrightarrow} \subseteq / \mathcal{M}$ and the left adjoint $p_{*}$ is the left Kan extension along $p$ followed by sheafification.

Proof For first statement it suffices to observe that $P(\widetilde{C} \text {, (Srpd })_{L}$ is right proper [11, Corollary 5.8]. $L$ is composition with $p$ and $R$ is pullback by $p$.

The derived functor $\underline{R}$ is just the pullback when applied to fibrant objects, and the pullback of a fibration with discrete fibers is also one. Furthermore the sections of $F \times{ }_{\mathcal{M}} \mathcal{M}^{\prime}(X)$ over $a \in \mathcal{M}^{\prime}(X)$ are exactly the sections of $F(X)$ over $p(a) \in \mathcal{M}(X)$, 
and so $\underline{R}$ agrees with $p^{*}$ when applied to the image of a sheaf on $\mathcal{M}$. It follows that $p^{*}$ is full and faithful and it remains to show that $p^{*}$ is essentially surjective.

The functor assigning to $\widetilde{\mho}^{\prime} \stackrel{f}{\longrightarrow} \mathcal{M}^{\prime}$ the second map in the factorization of $p \circ f$

$$
\mathfrak{F}^{\prime} \stackrel{\sim}{\longrightarrow} \mathfrak{F} \rightarrow \mathcal{M}
$$

as trivial cofibration followed by a fibration provides a model for the derived functor $\underline{L}$.

Given a fibration $\widetilde{\mho}^{\prime} \stackrel{f}{\longrightarrow} \mathcal{M}^{\prime}$ we have the following commutative diagram

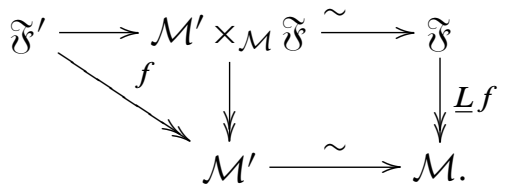

By two out of three the map $\widetilde{\mho}^{\prime} \rightarrow \mathcal{M}^{\prime} \times \mathcal{M} \mathfrak{\mho}$ is a weak equivalence between fibrant objects and is therefore a levelwise weak equivalence.

Given a sheaf $F^{\prime}$ on $\mathcal{M}^{\prime}$, applying $\underline{L}$ to $B F^{\prime} \stackrel{f}{\longrightarrow} \mathcal{M}^{\prime}$ yields a fibration $\mathfrak{\wp} \rightarrow \mathcal{M}$. The fiber of $\widetilde{r} \rightarrow \mathcal{M}$ over $X \rightarrow \mathcal{M}$ is

$$
\begin{aligned}
\operatorname{Hom}_{P(\mathfrak{S},(\mathfrak{s} p d) / \mathcal{M}}(X, \mathfrak{F}) & =\operatorname{hHom}_{P(\mathfrak{S},(\mathfrak{s} r p d) / \mathcal{M}}(X, \mathfrak{F}) \\
& \simeq \operatorname{hHom}_{P\left(\mathfrak{S},(\mathfrak{s} p d) / \mathcal{M}^{\prime}\right.}\left(\mathcal{M}^{\prime} \times_{\mathcal{M}} X, \mathcal{M}^{\prime} \times_{\mathcal{M}} \mathfrak{F}\right)
\end{aligned}
$$

where the equivalence arises from $R$ being part of a Quillen equivalence. Since $\mathcal{M}^{\prime} \times \mathcal{M} \mathfrak{\wp} \rightarrow \mathcal{M}^{\prime}$ is a fibration with homotopically discrete fibers the homotopy function complex of maps from any object in $P\left(\widetilde{C}\right.$, (5rpd) $/ \mathcal{M}^{\prime}$ into it is homotopically discrete. It follows that $\mathfrak{F} \rightarrow \mathcal{M}$ also has homotopically discrete fibers.

Given $\mathfrak{\Im} \rightarrow \mathcal{M}$ a fibration with levelwise homotopically discrete fibers a variation on the construction in the last proof can be used to construct a factorization $\mathfrak{\wp} \rightarrow$ $\widetilde{r}^{\prime} \rightarrow \mathcal{M}$, where $\widetilde{\wp} \rightarrow \mathcal{M}$ is a levelwise fibration with discrete fibers and $\widetilde{\wp} \rightarrow \widetilde{\wp}^{\prime}$ is a levelwise trivial fibration. It follows that $\mathfrak{F}^{\prime} \rightarrow \mathcal{M}$ is a fibration $P(\mathfrak{S} \text {, (5rpd })_{L}$ from the characterization of fibrations in Proposition 4-2. We conclude that $\mathfrak{\wp} \rightarrow \mathcal{M}$ is isomorphic to a sheaf in $\operatorname{Ho}(P(\widetilde{C}$, ( $r$ rpd $) / \mathcal{M})$. The pullback of $\mathfrak{s} \rightarrow \mathcal{M}$ is weakly equivalent to $B F^{\prime}$ and so $p^{*}: \operatorname{Sh}(\mathcal{M}) \rightarrow \operatorname{Sh}\left(\mathcal{M}^{\prime}\right)$ is essentially surjective.

Finally the description given for $p_{*}$ follows as it is the left adjoint of $p^{*}$.

Remark 4-9 Since sheaves of abelian groups are just abelian group objects in the category of sheaves Theorem 4-7 also yields an equivalence of sheaves of abelian groups. Similarly we obtain equivalences of sheaves of rings, simplicial sets, and have 
the following equivalence for sheaves of modules (see Mac Lane and Moerdijk [15, page 95]).

Corollary 4-10 Let $\mathcal{M}^{\prime} \stackrel{p}{\longrightarrow} \mathcal{M}$ be a weak equivalence in $P\left(\mathfrak{S}^{5} \text {, (Srpd }\right)_{L}$. Let $\mathcal{O}$ be a sheaf of rings on $\mathcal{M}$, and let $\mathcal{O}^{\prime}=p^{*} \mathcal{O}$, then $p^{*}$ induces an equivalence of categories $(\mathcal{O}-$ mod $) \stackrel{p^{*}}{\longrightarrow}\left(\mathcal{O}^{\prime}-m o d\right)$.

I learned the following definition from M Hopkins ${ }^{1}$.

Definition 4-11 Let $\mathcal{O}$ be a sheaf of rings on $\mathcal{M}$. A quasi-coherent sheaf of modules relative to $\mathcal{O}$ is an $\mathcal{O}$ module $\mathfrak{\&}$ which is locally presentable. This means that for every $X \stackrel{a}{\longrightarrow} \mathcal{M}$ there exists a cover $\left\{U_{i} \stackrel{u_{i}}{\longrightarrow} X\right\}$ in $\mathfrak{S}$ and exact sequences of $\left(a \circ u_{i}\right)^{*} \mathcal{O}_{-}$ modules

$$
\oplus_{I}\left(a \circ u_{i}\right)^{*} \mathcal{O} \rightarrow \oplus_{J}\left(a \circ u_{i}\right)^{*} \mathcal{O} \rightarrow\left(a \circ u_{i}\right)^{*} \mathfrak{\wp} \rightarrow 0 .
$$

The category $\mathcal{O}-\bmod _{q c}$ of quasi-coherent modules is by definition the full subcategory of $\mathcal{O}-\bmod$ whose objects are quasi-coherent sheaves. This is not necessarily an abelian category. Even if it is an abelian category, the inclusion of $\mathcal{O}-\bmod _{q c}$ in $\mathcal{O}-\bmod$ is not necessarily exact. See Section 5.4 for a discussion of this in the case of affine schemes in the flat topology.

Corollary 4-12 Let $\mathcal{O}$ be a sheaf of rings on $\mathcal{M}$. A weak equivalence $\mathcal{M}^{\prime} \stackrel{p}{\longrightarrow} \mathcal{M}$ in $P\left(\subseteq,(S r p d)_{L}\right.$ induces an equivalence of categories between quasi-coherent $\mathcal{O}$ modules and quasi-coherent $p^{*} \mathcal{O}$ modules.

Proof It is obvious that $p^{*}$ applied to a quasi-coherent $\mathcal{O}$ module is a quasi-coherent $p^{*} \mathcal{O}$ module.

Conversely, let $M$ be an $\mathcal{O}$ module such that $p^{*} M$ is a quasi-coherent $p^{*} \mathcal{O}$ module. Given $X \stackrel{a}{\longrightarrow} \mathcal{M}$, it follows from the local lifting conditions that we can find a cover of the form $(U, p(b)) \stackrel{(u, \alpha)}{\longrightarrow}(X, a)$ such that $p(b)^{*} M$ is presentable. The isomorphism $p(b) \stackrel{\alpha}{\longrightarrow} a \circ u$ induces a natural isomorphism between the functors $(p(b))^{*}$ and $(a \circ u)^{*}$ from sheaves of rings on $\mathcal{M}$ to sheaves of rings on $U$. It follows that $(p(b))^{*} \mathcal{O} \cong(a \circ u)^{*} \mathcal{O}$ and that there is an equivalence of categories between quasicoherent modules over $(p(b))^{*} \mathcal{O}$ and $(a \circ u)^{*} \mathcal{O}$. Since $(p(b))^{*} M=b^{*} p^{*} M$ is quasi-coherent $(a \circ u)^{*} M$ is also quasi-coherent and hence so is $M$.

We note that while $p^{*}$ is always exact as a functor between categories of $\mathcal{O}$ modules, it will not in general be exact when restricted to $\mathcal{O}-\bmod _{q c}$.

\footnotetext{
${ }^{1}$ It follows from faithfully flat descent (see Section 5.4) that this definition generalizes [14, Definition 13.2.2] for the étale site of a Deligne-Mumford stack. Roughly speaking the difference between the definitions is that [14] requires a sheaf to be globally presentable, in the sense that for each $X \rightarrow \mathcal{M}$ the sheaf has what we have called a presentation, while we only require this to hold locally.
} 


\subsection{Application: sheaf cohomology spectral sequence}

If $\mathfrak{F}$ is a sheaf of abelian groups on $\mathcal{M}$ we can regard $\mathfrak{\wp}$ as an abelian group object in $P(\mathfrak{C}$, (Srpd $) / \mathcal{M}$. The global sections $\Gamma(\mathfrak{F})$ are isomorphic to the discrete simplicial

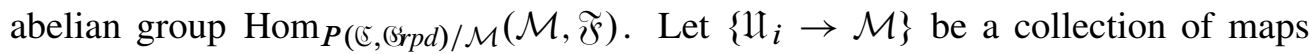
such that the induced map $|\mathfrak{H} \bullet| \rightarrow \mathcal{M}$ is a weak equivalence. Then we have weak equivalences of simplicial abelian groups

$$
\begin{aligned}
& \operatorname{Hom}_{\mathcal{M}}(\mathcal{M}, \mathfrak{\Im}) \cong \operatorname{Hom}_{\mathcal{M}}\left(\left|\mathcal{H}_{\bullet}\right|, \mathfrak{\Im}\right) \cong \lim \operatorname{Hom}_{\mathcal{M}}(\mathfrak{H} \bullet, \mathfrak{\Im}) \\
& \cong \lim \operatorname{Hom}_{\mathfrak{U}_{i}}\left(\mathfrak{U}_{i}, \mathfrak{\Im}_{\times_{\mathcal{M}}} \mathfrak{U}_{i}\right) \cong \lim \Gamma\left(\mathfrak{\Im}_{\mathcal{M}_{\mathcal{M}}} \mathfrak{U}_{i}\right) \text {. }
\end{aligned}
$$

The Grothendieck spectral sequence for composition of functors in this case yields a spectral sequence with $E_{2}$-term

$$
\check{H}^{i}\left(R^{j} \Gamma\left(\mathfrak{\mho} \times_{\mathcal{M}} \mathfrak{U}_{i}\right)\right) \Rightarrow R^{i+j} \Gamma(\mathfrak{\mho}) .
$$

This a generalization of the usual Čech cohomology spectral sequence for a cover which holds by the usual proof (see Tamme [22, Theorem I.3.4.4]).

\section{Descent for Sheaves on $\mathcal{M}$}

In this section we use the homotopy theory of categories recalled in Section 2.1 and a notion of homotopy decomposition of a site to prove descent statements for categories of sheaves on $\mathcal{M} \in P(\widetilde{C} \text {, (Srpd })_{L}$. A very special case of these statements yields a characterization of sheaves (of quasi-coherent modules) on the stack associated to a groupoid object in 5 .

In the case of affine schemes in the flat topology $\mathfrak{A} f f f_{\text {flat }}$ this says that quasi-coherent sheaves on the stack associated to a Hopf algebroid $(A, \Gamma)$ is equivalent to the category of $(A, \Gamma)$-comodules. Combining this result with Theorem 4-7 gives an alternate proof of a generalized change of rings theorem due to Mark Hovey [13].

\subsection{Descent}

In order to phrase our descent statement for categories of sheaves we need the following definition.

Definition 5-1 Let $T$ be a site. A homotopy decomposition of $T$ is an $I$ diagram of sites $T_{I}$ and an equivalence hocolim $T_{i} \stackrel{\sim}{\longrightarrow} T$ such that

(1) the induced maps $T_{i} \rightarrow T$ are maps of sites, 
(2) the images in $T$ of all the covers in $T_{i}$ generate the topology.

Proposition 5-2 Let $\mathfrak{D}$ be any category with products. A homotopy decomposition hocolim $T_{i} \stackrel{\sim}{\longrightarrow} T$ induces equivalences of categories

$$
\begin{gathered}
P(T, \mathfrak{D}) \stackrel{\sim}{\longrightarrow} \operatorname{holim} P\left(T_{i}, \mathfrak{D}\right) \\
\operatorname{Sh}(T, \mathfrak{D}) \stackrel{\sim}{\longrightarrow} \operatorname{holim} \operatorname{Sh}\left(T_{i}, \mathfrak{D}\right)
\end{gathered}
$$

Proof Even though $\mathfrak{D}$ is not necessarily a small category, the functor categories are well defined and the duality of our presentations of hocolim and holim in the previous subsection imply

$$
\left(\mathfrak{D}^{\mathrm{op}}\right)^{\operatorname{hocolim} T_{i}}=\operatorname{holim}\left(\mathfrak{D}^{\mathrm{op}}\right)^{T_{i}}
$$

for any $I$ diagram of categories $T_{I}$ and any category $\mathfrak{D}$.

The proof for sheaves is an easy application of the following lemma applied to the cosimplicial replacements of our diagrams.

Lemma 5-3 Let $\mathfrak{D}^{\bullet} \rightarrow \mathfrak{C}^{\bullet}$ be a map of cosimplicial categories such that each $\mathfrak{T}^{i} \rightarrow \mathfrak{S}^{i}$ is a full subcategory, then

$$
\operatorname{Tot}\left(\mathfrak{D}^{\bullet}\right)=\mathfrak{D}^{0} \times_{\Im^{0} 0} \operatorname{Tot}\left(\mathfrak{C}^{\bullet}\right) .
$$

In particular, if $\mathfrak{C} \stackrel{\sim}{\longrightarrow} \operatorname{Tot}\left(\mathfrak{C}^{\bullet}\right)$ and $\mathfrak{D} \hookrightarrow \mathfrak{C}$ is the full subcategory consisting of objects whose images in $\mathfrak{S}^{0}$ lie in the subcategory $\mathfrak{D}^{0}$ then $\stackrel{\mathfrak{D}}{\sim} \operatorname{Tot}\left(\mathfrak{D}^{\bullet}\right)$.

Proposition 5-4 Let $\mathfrak{H}_{I}$ be an $I$ diagram in $P(\mathfrak{S}$, ( Grpd). There is a canonical homotopy decomposition

$$
\operatorname{hocolim}\left(\mathfrak{S} / \mathfrak{U}_{i}\right) \stackrel{\sim}{\longrightarrow} \mathfrak{S} /\left(\operatorname{hocolim} \mathfrak{U}_{i}\right) .
$$

Proof Recall that $\mathfrak{E} / \mathfrak{U}_{i}$ is the Grothendieck construction on the functor $\mathfrak{U}_{i}$ : $\widetilde{5}^{\text {op }} \rightarrow$ (rrpd, or the coend $\mathfrak{S} /(-) \otimes \mathfrak{C} \mathfrak{U}_{i}$. An $I$ diagram $\mathfrak{H}_{I}$ in $P(\mathfrak{S}$, (Srpd) is a functor Crop $^{\mathrm{o}} \times I \rightarrow$ Gŕrpd. Since coends commute we have

$$
\begin{aligned}
\operatorname{hocolim}\left(\mathfrak{S} / U_{i}\right) & =\left(\mathfrak{S} /(-) \otimes \mathfrak{c} \mathfrak{U}_{I}\right) \otimes_{I} \pi_{\mathrm{oid}}(-/ I) \\
& \cong \mathfrak{S} /(-) \otimes \mathfrak{s}\left(\mathfrak{U}_{I} \otimes_{I} \pi_{\mathrm{oid}}(-/ I)\right)=\mathfrak{S} /\left(\text { hocolim } \mathfrak{U}_{i}\right) .
\end{aligned}
$$

Using the presentation of the homotopy colimit obtained by simplicial replacement of the diagram, we see that all maps $X \rightarrow\left(\right.$ hocolim $\mathfrak{U}_{i}$ ) factor through some $\mathfrak{U}_{i}$. It follows from Proposition 3-7 that the equivalence is a homotopy decomposition.

Algebraic ${ }^{3} \mathcal{G}$ Geometric Topology, Volume 7 (2007) 
The main descent statement of this section is the following corollary of the previous proposition.

Proposition 5-5 Let $\mathfrak{D}$ be any category with products, $\mathfrak{U}_{I}$ be an $I$ diagram in $P\left(\mathfrak{S}, \text { (ro r }^{\prime}\right)_{L}$ and hocolim $\mathfrak{U}_{i} \stackrel{\sim}{\longrightarrow} \mathcal{M}$ a weak equivalence. There is an equivalence of categories of presheaves

$$
P(\mathfrak{C} / \mathcal{M}, \mathfrak{D}) \stackrel{\sim}{\longrightarrow} \operatorname{holim} P\left(\mathfrak{C} / U_{i}, \mathfrak{D}\right)
$$

and sheaves

$$
\operatorname{Sh}(\mathfrak{S} / \mathcal{M}, \mathfrak{D}) \stackrel{\sim}{\longrightarrow} \operatorname{holim} \operatorname{Sh}\left(\mathfrak{S} / U_{i}, \mathfrak{D}\right)
$$

The previous result yields the following more explicit description of the category of sheaves on $\mathcal{M}$.

Corollary 5-6 Let $\mathfrak{U}_{I}$ be an I diagram in $P(\widetilde{C} \text {, (5rpd })_{L}$ and hocolim $\mathfrak{U}_{i} \stackrel{\sim}{\longrightarrow} \mathcal{M}$ a weak equivalence. The category of $\operatorname{Sh}(\mathcal{M})$ is equivalent to the category whose

- objects are collections $\left\{F_{i}, \alpha_{f}\right\}$ where

(i) $F_{i}$ is a sheaf on $\mathfrak{U}_{i}$,

(ii) $\alpha_{f}: f^{*} F_{j} \rightarrow F_{i}$ is an isomorphism, satisfying $\alpha_{\mathrm{id}_{i}}=\mathrm{id}_{F_{i}}$ and $\alpha_{g \circ f}=\alpha_{f} \circ f^{*}\left(\alpha_{g}\right)$ for each $i \in$ ob $I$ and $i \stackrel{f}{\longrightarrow} j \stackrel{g}{\longrightarrow} k$ $\in I$,

- morphisms $\left\{F_{i}, \alpha_{f}\right\} \rightarrow\left\{F_{i}^{\prime}, \beta_{f}\right\}$ are maps $\phi_{i}: F_{i} \rightarrow F_{i}^{\prime} \in \operatorname{Sh}\left(\mathfrak{U}_{i}\right)$ such that $\phi_{i} \circ \alpha_{f}=\beta_{f} \circ f^{*} \phi_{j}$.

\subsection{Descent for Quasi-coherent Sheaves}

Next we prove a version of these results for quasi-coherent sheaves.

Definition 5-7 A ringed space in $P\left(\mathfrak{S}\right.$, (كrrpd) is a pair $\left(\mathfrak{H}, \mathcal{O}_{\mathfrak{l}}\right)$ where $\mathfrak{U} \in P(\mathfrak{S}$, ( Srrpd) and $\mathcal{O}_{\mathfrak{l}}$ is a sheaf of rings on $\mathfrak{U}$. A morphism of ringed spaces in $P(\mathfrak{S}$, (Śrpd), $\left(\mathfrak{H}, \mathcal{O}_{\mathfrak{I}}\right) \rightarrow\left(\mathfrak{B}, \mathcal{O}_{\mathfrak{B}}\right)$ consists of a morphism $f: \mathfrak{U} \rightarrow \mathfrak{B} \in P(\mathfrak{C}$, (brpd $)$ and an isomorphism $\mathcal{O}_{\mathfrak{l}} \stackrel{\sim}{\longrightarrow} f^{*} \mathcal{O}_{\mathfrak{B}}$ of sheaves of rings on $\mathfrak{U}$.

Example 5-8 If $\mathfrak{S}=\mathfrak{U} f f$ with any reasonable topology and $\mathcal{M} \in P(\mathfrak{S}$, ( $r$ rpd $)$, the assignment $\mathcal{O}_{\mathcal{M}}(\operatorname{Spec} R \rightarrow \mathcal{M})=R$ yields a ringed space. 
An $I$ diagram of ringed spaces $\left(\mathfrak{H}_{I}, \mathcal{O}_{I}\right)$ consists of an $I$ diagram $\mathfrak{H}_{I}$ in $P(\mathfrak{C}$, ( Srrpd) together with sheaves of rings $\mathcal{O}_{i}$ on $\mathfrak{U}_{i}$ and for each $i \stackrel{\phi}{\longrightarrow} j$ isomorphisms $\phi^{*} \mathcal{O}_{j} \stackrel{\sim}{\longrightarrow}$ $\mathcal{O}_{i}$ of sheaves of rings on $\mathfrak{U}_{i}$ satisfying descent (that is, the conditions in Corollary $5-6)$. Such a diagram gives rise to an $I^{\mathrm{op}}$ diagram of categories

$$
i \mapsto \mathcal{O}_{i}-\bmod
$$

which assigns to a morphism $i \stackrel{\phi}{\longrightarrow} j \in I$ the composite functor

$$
\mathcal{O}_{i}-\bmod \longrightarrow \phi^{*} \mathcal{O}_{i}-\bmod \longrightarrow \mathcal{O}_{j}-\bmod .
$$

A diagram of ringed spaces $\left(\mathfrak{H}_{I}, \mathcal{O}_{I}\right)$ yields an element $\left[\mathcal{O}_{I}\right] \in \operatorname{holim}\left(\operatorname{Sh}\left(\mathfrak{H}_{i}, \Re\right.\right.$ ing $\left.)\right)$. Using Corollary 5-6 one can see that $\left[\mathcal{O}_{I}\right]$ is a ring object in $\operatorname{holim}\left(\operatorname{Sh}\left(\mathfrak{H}_{i}\right.\right.$, $\left.\left.\widetilde{S}_{e t}\right)\right)$ and it is straightforward to check that the category of modules over $\left[\mathcal{O}_{I}\right]$ is equivalent to the homotopy inverse limit of the $I^{\text {op }}$ diagram of categories $i \mapsto \mathcal{O}_{i}-\bmod$. As a consequence we have the following result.

Proposition 5-9 Let $\left(\mathcal{O}_{I}, \mathfrak{H}_{I}\right)$ be an I diagram of ringed spaces in $P(\mathfrak{S}$, (Srpd). Let $\mathcal{O}$ be a sheaf of rings on hocolim $\mathfrak{U}_{i}$ which is isomorphic to $\left[\mathcal{O}_{I}\right] \in \operatorname{holim} \operatorname{Sh}\left(\mathfrak{H}_{i}, \Re_{\text {ing }}\right)$. Then

$$
\mathcal{O}-\bmod \stackrel{\sim}{\longrightarrow} \operatorname{holim}\left(\mathcal{O}_{i}-\bmod \right)
$$

and this equivalence restricts to an equivalence for quasi-coherent modules

$$
\mathcal{O}-\bmod _{q c} \stackrel{\sim}{\longrightarrow} \operatorname{holim}\left(\mathcal{O}_{i}-\bmod _{q c}\right) .
$$

Proof Since $\operatorname{Sh}\left(\right.$ hocolim $\mathfrak{U}_{i}$, ธet $) \stackrel{\sim}{\longrightarrow}$ holim $\operatorname{Sh}\left(\mathfrak{U}_{i}\right.$, ๔et) the categories of modules over the ring objects $\mathcal{O}$ and $\left[\mathcal{O}_{I}\right]$ are equivalent, and the category of modules over $\left[\mathcal{O}_{I}\right]$ is equivalent to holim $\left(\mathcal{O}_{i}-\right.$ mod $)$. The proof for quasi-coherent modules follows by an application of Lemma 5-3.

\subsection{Descent for $\mathcal{M}_{\left(X_{0}, X_{1}\right)}$}

A groupoid object $\left(X_{0}, X_{1}\right) \in \mathfrak{S}$ determines a simplicial diagram in 5 :

$$
\cdots X_{1} \times_{X_{0}} X_{1} \times_{X_{0}} X_{1} \Longrightarrow X_{1} \times_{X_{0}} X_{1} \underset{p_{2}}{\stackrel{p_{1}, \mu}{\Longrightarrow}} X_{1} \stackrel{d}{\stackrel{d}{\rightleftharpoons}} X_{0}
$$

and therefore a simplicial diagram in $P(\mathfrak{S}$, ( $r p d)$ which we denote by $\left(X_{0}, X_{1}\right) \bullet$ By definition, the presheaf of groupoids represented by $\left(X_{0}, X_{1}\right)$ is the geometric realization of this simplicial diagram and so there is a weak equivalence

$$
\left|\left(X_{0}, X_{1}\right) \bullet\right| \stackrel{\sim}{\longrightarrow} \mathcal{M}_{\left(X_{0}, X_{1}\right)}
$$

Algebraic ${ }^{3} \mathcal{G}$ Geometric Topology, Volume 7 (2007) 
Using the model for the homotopy limit of a cosimplicial diagram given by $\operatorname{Tot}^{2}$ we see that an instance of Proposition 5-5 is the following result.

Corollary 5-10 The category of sheaves on a $\mathcal{M}_{\left(X_{0}, X_{1}\right)}$ is equivalent to the category with

(1) objects $(F, \alpha)$ with $F$ a sheaf on $X_{0}$ and $\alpha: d^{*} F \rightarrow r^{*} F$ an isomorphism satisfying $i^{*}(\alpha)=\mathrm{id}_{F}$ and $p_{2}^{*}(\alpha) \circ p_{1}^{*}(\alpha)=\mu^{*}(\alpha)$,

(2) morphisms the maps of sheaves $\phi: F \rightarrow F^{\prime}$ on $X_{0}$ satisfying $r^{*}(\phi) \circ \alpha=$ $\alpha^{\prime} \circ d^{*}(\phi)$.

Similarly, given a sheaf of rings $\mathcal{O}$ on $\mathcal{M}$, let $\mathcal{O}_{0}$ be the pullback of $\mathcal{O}$ to $X_{0}$. The category of quasi-coherent $\mathcal{O}$-modules is equivalent to the category with objects $(F, \alpha)$ with $F$ a quasi-coherent $\mathcal{O}_{0}$-module and $\alpha: d^{*} F \rightarrow r^{*} F$ an isomorphism of $d^{*} \mathcal{O}_{0-}$ modules (where $r^{*} F$ is regarded as a $d^{*} \mathcal{O}_{0}$-module via the canonical isomorphism $\left.d^{*} \mathcal{O}_{0} \simeq r^{*} \mathcal{O}_{0}\right)$ satisfying the relations above.

\subsection{Quasi-Coherent Sheaves on a Hopf Algebroid}

In this section $\subseteq$ is the category affine schemes (and all morphisms between them) with the flat topology. A groupoid object ( $\operatorname{Spec} A, \operatorname{Spec} \Gamma$ ) in $\mathfrak{Y} f f_{\text {flat }}$ is called a Hopf algebroid.

Given $\mathcal{M} \in P\left(\mathfrak{Q}\left(f f_{\text {flat }}\right.\right.$, Srrpd $)$ there is a natural choice of "structure sheaf" of rings $\mathcal{O}_{\mathcal{M}}$ defined by

$$
\mathcal{O}_{\mathcal{M}}(\operatorname{Spec} R, a)=R \text {. }
$$

For the rest of this section quasi-coherent sheaves will always refer to quasi-coherent modules relative to this structure sheaf.

In the site $\mathfrak{Y} f f_{\text {flat }} / \operatorname{Spec}(R)$ faithfully flat descent of modules (see Milne [16, Remark I.2.19]) tells us that quasi-coherent modules are not only locally presentable, but globally presentable. We include the argument for completeness.

Lemma 5-11 The category quasi-coherent sheaves on $\mathfrak{Y} f f_{\text {flat }} / \operatorname{Spec}(R)$ is equivalent to the opposite category of $R$-modules.

Proof Since $\otimes$ is right exact there is a functor from $R$-modules to quasi-coherent sheaves sending $M \rightarrow F_{M}$ where

$$
F_{M}\left(\operatorname{Spec} R^{\prime}\right)=M \otimes_{R} R^{\prime}
$$


It is clear that this functor is full and faithful. Given a quasi-coherent sheaf $\mathfrak{f}$ on $\mathfrak{A} f f_{\text {flat }} / \operatorname{Spec}(R)$ the definition of quasi-coherent implies that there is a cover $\left\{\operatorname{Spec} S_{i} \rightarrow \operatorname{Spec} R\right\}$ and $S_{i}$-modules $M_{i}$ so that

$$
\left.\mathfrak{\Im}\right|_{\mathfrak{A} f f / \operatorname{Spec}} S_{i} \cong M_{i} \otimes S_{i}(-)
$$

(since $\otimes$ is right exact). Evaluating $\mathfrak{\lessgtr}$ on $S_{i} \otimes_{R} S_{j}$ we see that

$$
M_{i} \otimes_{R} S_{j} \cong M_{j} \otimes_{R} S_{i} .
$$

$\mathfrak{\Im}(\operatorname{Spec} R)$ is the equalizer

$$
\prod M_{i} \rightrightarrows \prod_{i, j} M_{i} \otimes_{R} S_{j}
$$

Let $M$ be the value of this equalizer. Since $\operatorname{Spec} S_{i} \rightarrow \operatorname{Spec} R$ is a cover

$$
R \rightarrow \prod_{i} S_{i} \rightrightarrows \prod_{i, j} S_{i} \otimes_{R} S_{j}
$$

is exact on the left and remains so when we tensor with any $R$-module [16, Proposition I.2.7 and Remark I.2.19]. It follows that we can tensor equation (5-14) with $M_{k}$ and tensor (5-13) with $S_{k}$ to obtain the following isomorphisms

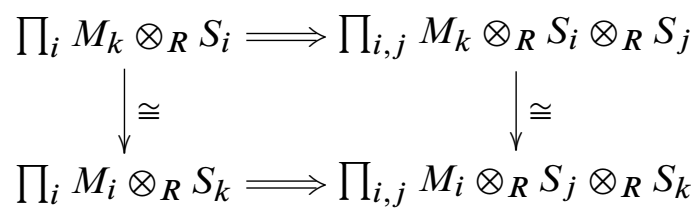

which induce an isomorphism between the equalizers $M \otimes_{R} S_{k} \cong M_{k}$. Given a map of rings $R \rightarrow R^{\prime}$, a similar argument shows that $\mathfrak{F}\left(\operatorname{Spec} R^{\prime}\right) \cong M \otimes_{R} R^{\prime}$, which completes the proof.

Proposition 5-15 The category of quasi-coherent sheaves on ( $\operatorname{Spec} A$, Spec $\Gamma$ ) (or $\left.\mathcal{M}_{(\operatorname{Spec} A, \operatorname{Spec} \Gamma)}\right)$ is equivalent to the category of comodules on the Hopf algebroid $(A, \Gamma)$.

Proof An Hopf algebroid $(A, \Gamma)$ yields a diagram

$$
A \stackrel{L}{\stackrel{R}{\longrightarrow}} \Gamma \Longrightarrow \Gamma \otimes_{A} \Gamma
$$

where the maps $\Gamma \rightarrow \Gamma \otimes_{A} \Gamma$ are $L \otimes 1_{\Gamma}, \mu$ and $1_{\Gamma} \otimes R$ with $\mu$ the comultiplication. By Corollary 5-10 and Lemma 5-11, a quasi-coherent sheaf consists of an $A$ module 
$M$ and an isomorphism of $\Gamma$-modules $\Gamma \otimes_{A} M \stackrel{\alpha}{\longrightarrow} M \otimes \Gamma$ making the following diagram commute:

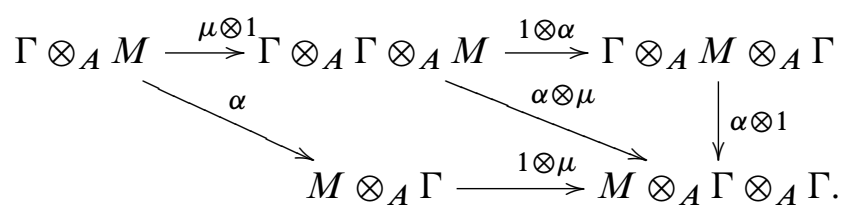

Let $\phi$ be defined as the composition

$$
M \stackrel{R \otimes 1}{\longrightarrow} \Gamma \otimes_{A} M \stackrel{\alpha}{\longrightarrow} M \otimes_{A} \Gamma .
$$

Precomposing the commutative diagram above with the map $M \stackrel{R \otimes 1}{\longrightarrow} \Gamma \otimes_{A} M$ and using the identity $1 \otimes R \otimes 1=\mu \otimes 1 \circ R \otimes 1$, one can see that the composition along the top and down to $M \otimes_{A} \Gamma \otimes_{A} \Gamma$ is $(\phi \otimes 1) \circ \phi$. The composition along the bottom is $(1 \otimes \mu) \circ \phi$. so $\phi$ defines a comodule structure on $M$ (see Ravenel [19, Appendix A.1]).

Conversely a comodule structure on $M$ is a map of $A$-bimodules $M \rightarrow M \otimes_{A} \Gamma$ and so there is an extension of this map over $M \stackrel{R \otimes 1}{\longrightarrow} \Gamma \otimes_{A} M$ providing a $\Gamma$-module isomorphism $\Gamma \otimes_{A} M \stackrel{\alpha}{\longrightarrow} M \otimes_{A} \Gamma$. Another diagram chase shows that the comodule identity is equivalent to the condition that $\alpha$ satisfies descent.

The previous result together with Corollary 4-12 yields the following result of Hovey [13, Theorems A and C].

Corollary 5-16 Let $\left(A, \Gamma_{A}\right)$ and $\left(B, \Gamma_{B}\right)$ be two Hopf algebroids, for which (Spec $A$, $\left.\operatorname{Spec} \Gamma_{A}\right)$ and $\left(\operatorname{Spec} B, \operatorname{Spec} \Gamma_{B}\right)$ are weakly equivalent in $P\left(\mathfrak{Q} f f_{\text {flat }} \text {, (Srpd }\right)_{L}$. The category of $\left(A, \Gamma_{A}\right)$ comodules is equivalent to the category of $\left(B, \Gamma_{B}\right)$-comodules.

For the sake of completeness, we use the equivalence of categories of Proposition 5-15 to provide the reader with an example of a category of quasicoherent sheaves which is not an abelian category and conclude with some related remarks.

Example 5-17 Consider the Hopf algebroid $\left(\mathbb{Z}, \mathbb{Z}[\epsilon] /\left(p \epsilon, \epsilon^{2}\right)\right)$ where the composition

$$
\mathbb{Z}[\epsilon] /\left(p \epsilon, \epsilon^{2}\right) \rightarrow \mathbb{Z}\left[\epsilon_{1}\right] /\left(p \epsilon_{1}, \epsilon_{1}^{2}\right) \otimes_{\mathbb{Z}} \mathbb{Z}\left[\epsilon_{2}\right] /\left(p \epsilon_{2}, \epsilon_{2}^{2}\right)
$$

is given by sending $\epsilon$ to $\left(\epsilon_{1}+\epsilon_{2}+\epsilon_{1} \epsilon_{2}\right)$. Every abelian group $M$ has (at least) two comodule structures

$$
M \rightarrow \mathbb{Z}[\epsilon] /\left(p \epsilon, \epsilon^{2}\right) \otimes M
$$

Algebraic $8 \mathcal{G}$ Geometric Topology, Volume 7 (2007) 
one of which is given by $1 \otimes \mathrm{id}_{M}$ and the other by $(1+\epsilon) \otimes \mathrm{id}_{M}$. We denote these by $(M, 1)$ and $(M, 1+\epsilon)$ respectively. Consider the epimorphism $\left(\mathbb{Z} / p^{2}, 1\right) \stackrel{r}{\longrightarrow}(\mathbb{Z} / p, 1)$. There are monomorphisms

$$
(\mathbb{Z} / p, 1) \stackrel{i}{\longrightarrow}\left(\mathbb{Z} / p^{2}, 1\right), \quad \text { and } \quad(\mathbb{Z} / p, 1+\epsilon) \stackrel{i^{\prime}}{\longrightarrow}\left(\mathbb{Z} / p^{2}, 1\right)
$$

such that $r$ is the cokernel of both $i$ and $i^{\prime}$. Clearly $(\mathbb{Z} / p, 1)$ and $(\mathbb{Z} / p, 1+\epsilon)$ are not isomorphic but in an abelian category a monomorphism must be the kernel of its cokernel.

Even when the category of quasi-coherent $\mathcal{O}_{\mathcal{M}}$-modules is an abelian category, the inclusion into $\mathcal{O}_{\mathcal{M}}$-modules is not necessarily exact. An example to consider is the multiplication by $p$ map

$$
\mathcal{O}_{\mathrm{Spec} \mathbb{Z}} \stackrel{p}{\longrightarrow} \mathcal{O}_{\mathrm{Spec}} \mathbb{Z} .
$$

Let $K_{p}$ be the kernel of this map as an $\mathcal{O}_{\text {Spec } \mathbb{Z}}$ module. Then $K_{p}(\operatorname{Spec} R)$ is the $p$-torsion in $R$ and so $K_{p}$ is not quasi-coherent. The kernel of multiplication by $p$ within quasi-coherent modules exists and is 0 .

Furthermore, given a map Spec $R^{\prime} \stackrel{f}{\longrightarrow}$ Spec $R$, pullback of sheaves is an exact functor but pullback of quasi-coherent sheaves is not in general: the pullback of a quasi-coherent sheaf $F_{M}$ on $\operatorname{Spec} R$ is

$$
f^{*} F_{M}\left(\operatorname{Spec} R^{\prime}\right)=F_{M}\left(\operatorname{Spec} R^{\prime} \rightarrow \operatorname{Spec} R\right) \cong F_{M \otimes_{R} R^{\prime}}
$$

and therefore, for quasi-coherent sheaves, the pullback functor corresponds to the tensor product $(-) \otimes_{R} R^{\prime}$ which is not always exact.

\subsection{A different approach}

An alternate approach to the descent statements in this section and the homotopy invariance of the previous section would be to make use of the stack of sheaves, which we learned about from M Hopkins.

Disregarding set theoretic questions one can define a stack of sheaves $\subseteq \in P(\mathfrak{S}$, (Srpd) associating to $X \in \mathfrak{C}$ the groupoid of sheaves on $X$. In a similar fashion one can define

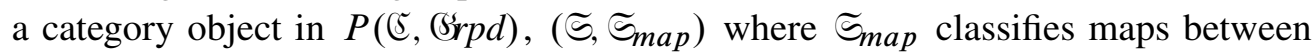
sheaves.

Given $\mathcal{M} \in P(\mathcal{S}$, (Srpd) one could then define $\operatorname{Sh}(\mathcal{M})=\operatorname{Hom}(\mathcal{M}, \subseteq)$. With this definition, a sheaf on $\mathcal{M}$ would consist of a compatible assignment of sheaves to each $X \in \mathfrak{S}$ and map $X \rightarrow \mathcal{M}$. The equivalence of this definition with our definition of 
sheaf on $\mathfrak{S} / \mathcal{M}$ should come down to the equivalence of $\widetilde{S} / \mathcal{M}$ with the homotopy colimit over $5 / \mathcal{M}$ of the categories $5 / X$.

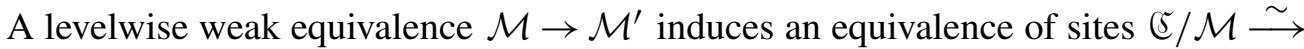
$5 / \mathcal{M}^{\prime}$ and hence of categories of sheaves. Since cofibrant replacement is a levelwise weak equivalence we would have

$$
\operatorname{Hom}(\mathcal{M}, \subseteq) \stackrel{\sim}{\longrightarrow} \operatorname{hHom}(\mathcal{M}, \subseteq) .
$$

An immediate corollary of this would be our homotopy invariance and descent results.

\section{References}

[1] M Artin, Versal deformations and algebraic stacks, Invent. Math. 27 (1974) 165-189 MR0399094

[2] M Behrens, A modular description of the K(2)-local sphere at the prime 3, Topology 45 (2006) 343-402 MR2193339

[3] P Deligne, D Mumford, The irreducibility of the space of curves of given genus, Inst. Hautes Études Sci. Publ. Math. (1969) 75-109 MR0262240

[4] D Dugger, S Hollander, D C Isaksen, Hypercovers and simplicial presheaves, Math. Proc. Cambridge Philos. Soc. 136 (2004) 9-51 MR2034012

[5] J Giraud, Cohomologie non abélienne, Grundlehren der mathematischen Wissenschaften 179, Springer, Berlin (1971) MR0344253

[6] P G Goerss, (Pre-)sheaves of ring spectra over the moduli stack of formal group laws, from: "Axiomatic, enriched and motivic homotopy theory", NATO Sci. Ser. II Math. Phys. Chem. 131, Kluwer Acad. Publ., Dordrecht (2004) 101-131 MR2061853

[7] PG Goerss, H-W Henn, M Mahowald, C Rezk, A resolution of the $K(2)$-local sphere at the prime 3, Ann. of Math. (2) 162 (2005) 777-822 MR2183282

[8] P G Goerss, M J Hopkins, Moduli spaces of commutative ring spectra, from: "Structured ring spectra", London Math. Soc. Lecture Note Ser. 315, Cambridge Univ. Press, Cambridge (2004) 151-200 MR2125040

[9] PS Hirschhorn, Model categories and their localizations, Mathematical Surveys and Monographs 99, American Mathematical Society, Providence, RI (2003) MR1944041

[10] S Hollander, Characterizing algebraic stacks, Proc. Amer. Math. Soc. (to appear)

[11] S Hollander, A homotopy theory for stacks, Israel J. Math. (to appear)

[12] M J Hopkins, Algebraic topology and modular forms, from: "Proceedings of the International Congress of Mathematicians, Vol. I (Beijing, 2002)", Higher Ed. Press, Beijing (2002) 291-317 MR1989190

Algebraic 83 Geometric Topology, Volume 7 (2007) 
[13] M Hovey, Morita theory for Hopf algebroids and presheaves of groupoids, Amer. J. Math. 124 (2002) 1289-1318 MR1939787

[14] G Laumon, L Moret-Bailly, Champs algébriques, Ergebnisse der Mathematik und ihrer Grenzgebiete 39, Springer, Berlin (2000) MR1771927

[15] S Mac Lane, I Moerdijk, Sheaves in geometry and logic, Universitext, Springer, New York (1994) MR1300636

[16] J S Milne, Étale cohomology, Princeton Mathematical Series 33, Princeton University Press, Princeton, N.J. (1980) MR559531

[17] N Naumann, Comodule categories and the geometry of the stack of formal groups arXiv:math.AT/0503308

[18] E Pribble, A Theory of Algebraic Stacks for Stable Homotopy Theory, the Algebraic Geometry of Formal Group Laws, and the Algebraic Chromatic Convergence Theorem, PhD thesis, Northwestern University (2004) Available at http:// www.math.rochester.edu/people/faculty/pribble/publications.html

[19] D C Ravenel, Complex cobordism and stable homotopy groups of spheres, Pure and Applied Mathematics 121, Academic Press, Orlando, FL (1986) MR860042

[20] C Rezk, A model category for categories, preprint

[21] C Rezk, Notes on the Hopkins-Miller theorem, from: "Homotopy theory via algebraic geometry and group representations (Evanston, IL, 1997)", Contemp. Math. 220, Amer. Math. Soc., Providence, RI (1998) 313-366 MR1642902

[22] G Tamme, Introduction to étale cohomology, Universitext, Springer, Berlin (1994) MR1317816Translated from the German by Manfred Kolster

Department of Mathematics, Hebrew University

Jerusalem, Israel

Center for Mathematical Analysis, Geometry, and Dynamical Systems, Instituto Superior Técnico

Universidade Técnica de Lisboa, Lisbon, Portugal

sjh@math.huji.ac.il

Received: 30 June 2006 Revised: 10 November 2006 